\title{
4,9-diaminoacridines and 4-aminoacridines as antiplasmodial dual-stage hits
}

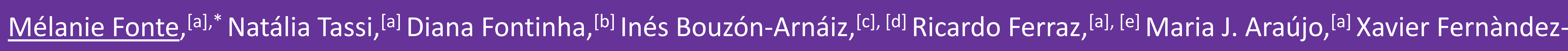
Busquets, ${ }^{[c], ~[d], ~[f] ~ M i g u e l ~ P r u d e ̂ n c i o, ~}{ }^{[b]}$ Paula Gomes, ${ }^{[a]}$ Cátia Teixeira ${ }^{[a]}$

a) LAQV-REQUIMTE, Departamento de Química e Bioquímica, Faculdade de Ciências, Universidade do Porto, Portugal; b) Instituto de Medicina Molecular, Faculdade de Medicina, Universidade de Lisboa, Portugal; c) Nanomalaria Group, Institute for Bioengineering of Catalonia (IBEC), The Barcelona Institute of Science and Technology, Spain; d) Barcelona Institute for Global Health (ISGlobal), Barcelona Center for International Health Research (CRESIB), Hospital Clínic-Universitat de Barcelona, Spain; e) Ciências Químicas e das Biomoléculas, Escola

\section{Aim}

Malaria is one of the deadliest infectious diseases in the world. The eradication of malaria has not yet been achieved, mainly due to the emergence of resistant parasites. Therefore, multi-target drugs have being prioritized in antimalarial drug discovery, as targeting more than one process in the Plasmodium life cycle is likely to increase efficiency, while decreasing the chances of emergence of resistance by the parasite. ${ }^{[1]}$ In this sense, and keeping in mind that the one costeffective strategy is to repurpose existing drugs for malaria, or to rescue antimalarial pharmacophores, ${ }^{[1]}$ we reported the synthesis and in vitro evaluation of two novels acridines families (4,9-diaminoacridines and 4-aminoacridines) though the combination of primaquine (PQ) and chloroquine (CQ), two well-known antimalarial drugs with activities in different stages of the parasite life cycle, ${ }^{[2]}$ hence acting as dual-stage antiplasmodial hits.
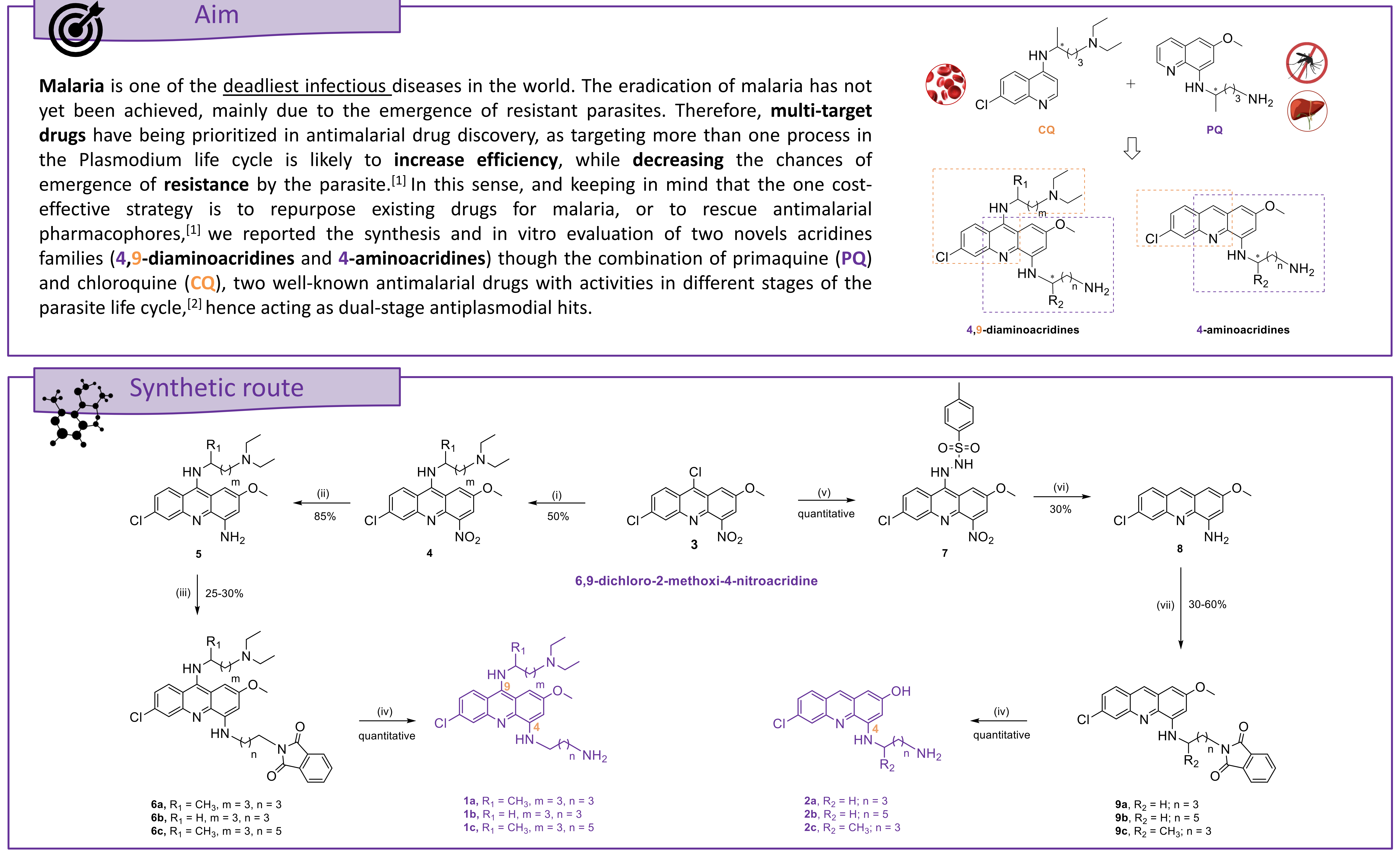

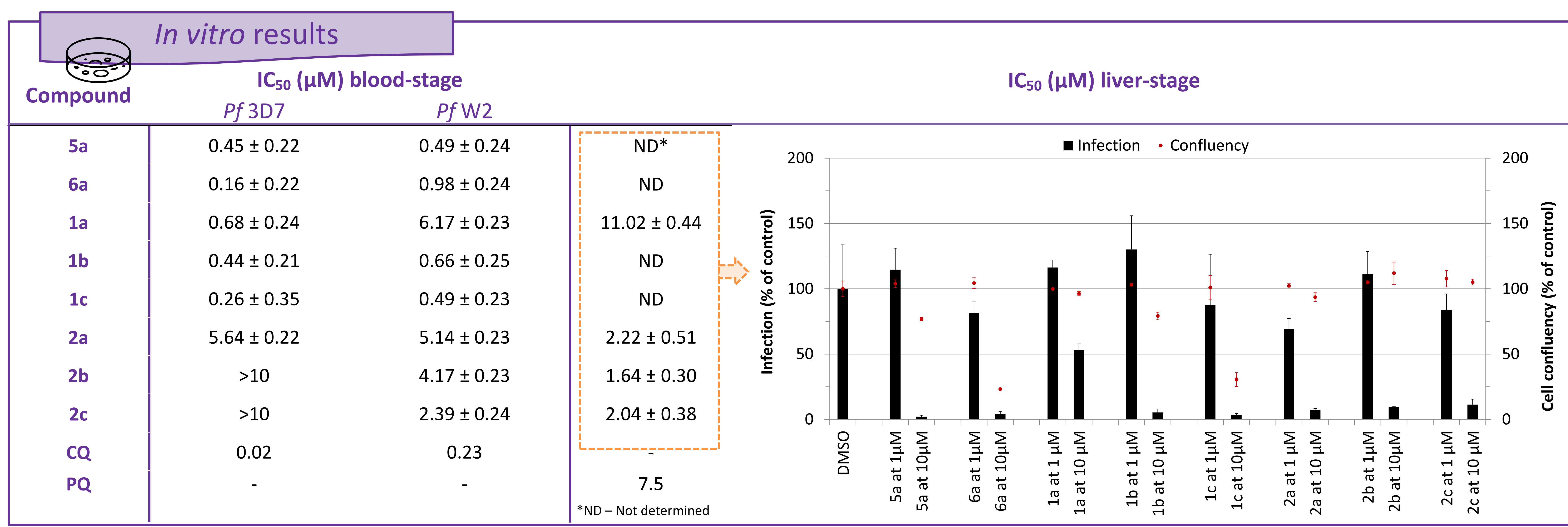

\section{Conclusions}

- Synthesized compounds retained the activity of the parent compounds, which makes them potential antimalarial dual-stage hits;

- Activity is dependent of the nature and length of side chain introduced in position 4 and 9 of the acridine ring;
References: [1] Teixeira, C. et al. Chem Rev. 2014, 114, 11164-11220; [2] Fonte, M. et al. Tetrahedron Lett., 2019, 60, 1166-1169.

Acknowledgement: This work was developed within the scope of projects UIDB/50006/2020 and PTDC/BTMSAL/29786/2017, financed by national funds through the Fundação para a Ciência e Tecnologia (FCT). MF thanks the FCT for the doctoral grant SFRH/BD/147345/2019. 